\title{
Combination of Biological Agents in Moderate to Severe Pediatric Inflammatory Bowel Disease: A Case Series and Review of the Literature
}

\author{
Christine Olbjørn ${ }^{1,2}$. Jon Bergreen Rove ${ }^{1}$ Jørgen Jahnsen ${ }^{2,3}$
}

Published online: 7 May 2020

(c) The Author(s) 2020

\begin{abstract}
Background Treatment with biological agents such as anti-tumor necrosis factors (TNFs) has become standard of care in moderate to severe pediatric inflammatory bowel disease (IBD). However, a significant proportion of patients experience loss of response to anti-TNFs, need treatment escalation, or develop side effects. There is no data in the literature regarding combination of biological agents in pediatric IBD.

Methods At our hospital, which is a tertiary referral center, we have combined the anti-TNF infliximab with either vedolizumab or ustekinumab in patients with severe pediatric IBD. The indications for dual biological therapy were insufficient efficacy of infliximab or vedolizumab monotherapy, or side effects such as psoriasis due to anti-TNFs.

Results Eight patients (four boys) aged 14-17.5 years received a combination of infliximab and vedolizumab due to only a partial response to infliximab, four with Crohn's disease (CD) and four with ulcerative colitis (UC). Clinical remission was achieved in four patients ( $3 \mathrm{UC}$ ) and four had a colectomy (3 CD, $1 \mathrm{UC}$ ). Five CD patients (3 girls) aged 11-17 years, on maintenance therapy with infliximab, developed psoriasis resistant to topical treatment. A combination of infliximab and ustekinumab resulted in clinical remission of CD without skin symptoms. No serious adverse events occurred in any of the patients on combination therapy. Thirteen publications report on combining biologicals, all in adult IBD.

Conclusion In pediatric IBD, combining biological agents seems to be safe and beneficial in selected patients. The safety should be addressed in long-term follow-up studies.
\end{abstract}

These cases were presented at ESPGHAN 2019 in Glasgow as a poster.

Christine Olbjørn

chrisolb@gmail.com

1 Department of Pediatric and Adolescent Medicine, Akershus University Hospital, 1478 Lørenskog, Norway

2 Institute of Clinical Medicine, University of Oslo, Oslo, Norway

3 Department of Gastroenterology, Akershus University Hospital, Lørenskog, Norway

\section{Key Points}

In pediatric inflammatory bowel disease patients, there are no publications on combining biological therapies.

We have treated eight patients with a combination of infliximab and vedolizumab, and five patients with infliximab in combination with ustekinumab in order to gain clinical remission or to treat side effects such as psoriasis caused by infliximab.

We experienced no serious adverse events and in nine of the 13 patients, clinical remission was achieved and the side effects managed with the combination of biologicals. 


\section{Introduction}

Pediatric inflammatory bowel disease (PIBD) is often aggressive with a high inflammatory burden at diagnosis and a complicated disease course [1]. Treatment with biological agents such as anti-tumor necrosis factors (TNFs) has improved the clinical outcome and has become standard of care in moderate to severe PIBD. However, patients develop side effects and up to $40 \%$ experience loss of response to anti-TNFs, which necessitates treatment escalation [2]. Psoriasis due to TNF blocker treatment is increasingly recognized in patients with IBD [3]. Considering that increased levels of TNF $\alpha$ play a key role in the pathogenesis of psoriasis, the occurrence under anti-TNF therapy seems to be paradoxical. New biological drugs for the treatment of IBD offer the possibility of combining agents that antagonize different pathways, potentially resulting in an additive effect for refractory disease and management of side effects. Vedolizumab (VDZ) and ustekinumab (UST) are such biological drugs, each with a different mode of action to anti-TNFs. These agents have demonstrated efficacy in IBD and can be used in patients failing or losing response to anti-TNFs [4-6].

VDZ is a humanized monoclonal antibody that specifically recognizes the $\alpha 4 \beta 7$ integrin receptor on lymphocytes and blocks the migration from the bloodstream to the intestinal mucosa, which reduces the white cell influx to inflamed tissue. This gut-selective mechanism of action differentiates it from the other biologics, which all have a more systemic influence on the immune system [7]. VDZ is not approved for pediatric patients, but use in PIBD has shown clinical efficacy with remission rates in ulcerative colitis (UC) and Crohn's disease (CD) reaching $76 \%$ and $42 \%$, respectively. The drug is well tolerated with a lack of serious adverse events [8-13].

UST is approved for the treatment of moderate-to-severe psoriasis in adolescents (aged 12 years and older) as well as for adult CD and UC [14-16]. UST is a human monoclonal antibody directed against the p40 subunit of interleukin (IL)-12 and IL-23 and exerts its anti-inflammatory effects by inhibiting these cytokines and their downstream pro-inflammatory signals, and inhibits Th1 and Th2 lymphocytes. Off-label use in the PIBD population is increasing and is reported to be safe and efficacious [17-19].

There are no publications on combining biological agents in PIBD, and data on combining biological agents with different modes of action in adult IBD is limited. We present our experience with combining biological agents in pediatric patients with IBD and give a review of the literature regarding the combination of biologicals in adult IBD patients.

\section{Methods}

\subsection{Case Reports}

We describe our experience with combining biological agents in PIBD between May 2014 and March 2020 in our hospital, a tertiary referral center and University Hospital.

PIBD patients who had insufficient response to infliximab (IFX) were treated with a combination of IFX and VDZ. Before adding VDZ, adequate drug levels of IFX were ensured with trough level measurements and IFX was optimized with dose or interval adjustments. Disease activity was assessed with fecal calprotectin, laboratory work up, endoscopy, and magnetic resonance imaging (MRI).

UST was added to IFX in patients who developed paradoxical psoriasis not responding to topical treatment. All patients were evaluated by a dermatologist. During the combination therapy we continuously screened the patients for adverse events.

\subsection{Literature Review}

A literature search of the PubMed database and Web of Science was performed using relevant terms and keywords related to combining biological drugs in the treatment of IBD.

\section{Results}

\subsection{Combination Therapy in Response to Insufficient Effect of Infliximab (IFX) or Vedolizumab (VDZ) Alone}

Eight patients (four boys and four girls, aged 14-17.5 years) received a combination of IFX and VDZ. Four of the patients had CD, four had UC (Table 1). They had been treated with IFX for a median of 2 years (range 4 months to 7 years), but experienced a flare that continued after optimizing IFX treatment. Before escalating therapy, active inflammation was confirmed endoscopically and histologically in all patients. Infectious gastroenteritis including Clostridium difficile, Cytomegalovirus, and Epstein Barr virus was excluded. Other treatment options-exclusive enteral nutrition (EEN) in $\mathrm{CD}$, immunomodulators (azathioprine or methotrexate), and corticosteroids-were tried without inducing remission. In the first two CD patients, we stopped IFX and switched to VDZ, but VDZ was not effective as monotherapy. One patient experienced a flare of both her CD and concomitant rheumatoid arthritis without IFX, the other CD patient developed a perianal fistula on VDZ monotherapy. In both patients, IFX was reintroduced after 4 and 5 months due 


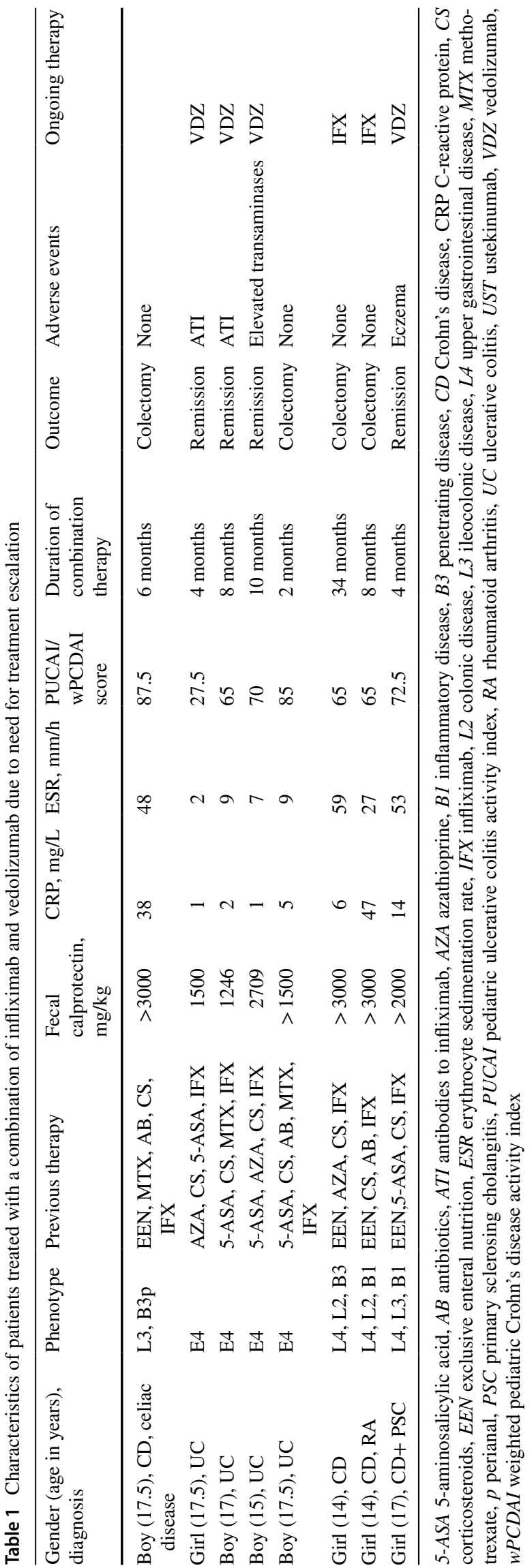

to lack of disease control. The remaining six patients had active disease and some effect of IFX, and after the experience with IBD flares after IFX discontinuation in the two previous patients we continued IFX infusions in six patients every 4-6 weeks while VDZ was initiated. Other immunomodulator therapy was stopped except in two patients who continued methotrexate. We used an induction scheme of VDZ $300 \mathrm{mg}$ intravenously in weeks $0,2,6$, and 10 , and continued infusions every 6-8 weeks, following trough level measurements. Three of the eight patients achieved complete clinical and biochemical remission on combination therapy, all three with UC. None of these patients were using methotrexate. After a mean combination treatment duration of 6.5 months (range 4-10 months), the patients were switched to monotherapy with VDZ; in two patients, this was due to the development of antibodies to IFX. They have both been in remission on VDZ monotherapy since, for more than 15 months. One UC patient developed elevated transaminases while on VDZ and IFX combination therapy, and IFX was stopped. This patient continued having slightly elevated transaminases, and is being evaluated for possible primary sclerosing cholangitis (PSC) and a liver biopsy is scheduled. His UC is in remission with VDZ monotherapy. One CD girl is in clinical remission, but has ongoing inflammation with an elevated erythrocyte sedimentation rate (ESR) and C-reactive protein (CRP). She has concomitant PSC. IFX was stopped after 4 months' combination therapy due to severe eczema on the face, scalp, and body. The eczema was thought to be due to IFX as it resolved after cessation of IFX. Altogether, four patients ( $3 \mathrm{CD}, 1 \mathrm{UC}$ ) had a colectomy due to failure of achieving remission with combination therapy. No infections or serious adverse events of the combination therapy were noted.

\subsection{Combining IFX and Ustekinumab (UST) in Response to Side Effects of IFX}

Five CD patients (three girls and two boys, aged 11-17 years), on maintenance therapy with IFX, developed severe paradoxical psoriasis resistant to topical treatment (Table 2). The psoriasis was mainly located in the periauricular region and on the scalp, with severe hair loss in three patients. One boy and one girl had additional palmoplantar psoriasis; another girl had concomitant anogenital psoriasis. These patients had been treated with IFX for a median of 2 years (range 7 months to 7 years) and were in clinical and biochemical remission with trough levels within target range $(5-8 \mu \mathrm{g} / \mathrm{mL})$ [20] (Table 3). In the first two patients with hair loss and scalp psoriasis, we switched treatment from IFX to subcutaneous UST $90 \mathrm{mg}$, with an induction regimen of weekly injections for 4 weeks, and every 8 weeks thereafter. The skin lesions and hair loss improved, but their CD flared with abdominal pain, diarrhea, weight 


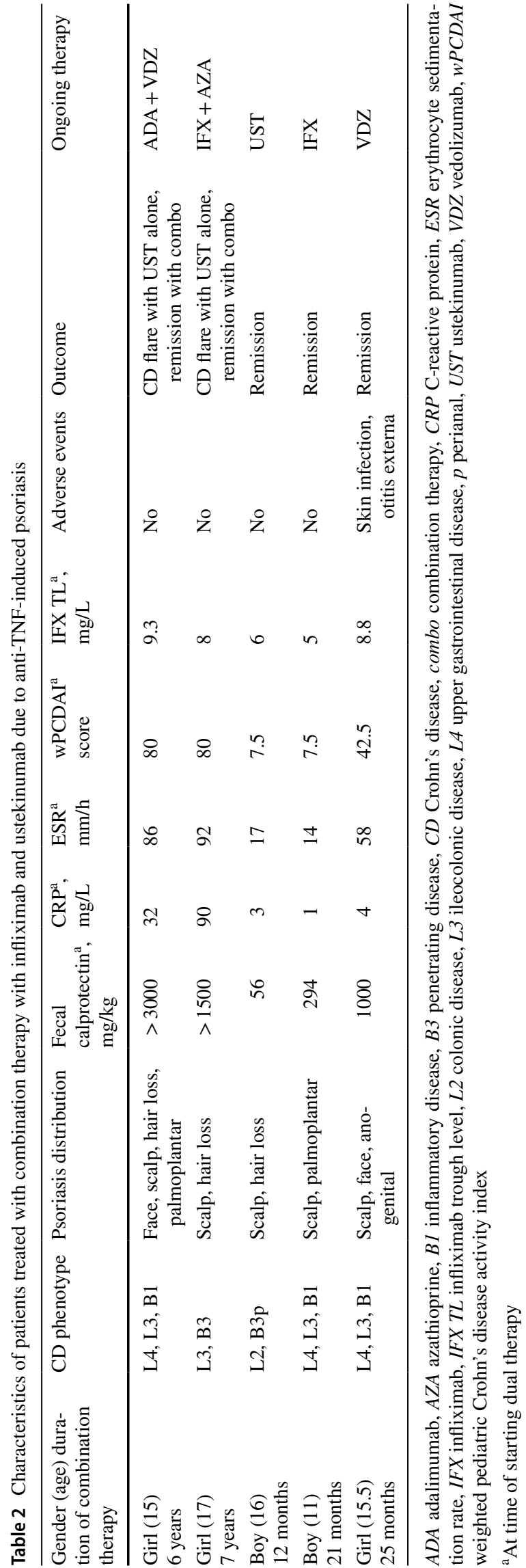

loss, and elevated fecal calprotectin levels. Reintroduction of IFX was initiated. With the combination of IFX every 6-8 weeks and UST every $8-12$ weeks, remission of CD was regained without re-occurrence of the psoriasis. One girl received combination treatment for 7 years and then continued with IFX monotherapy. On monotherapy she developed a rectovaginal fistula and abscess. She still has some psoriatic lesions, but no hair loss and the psoriasis is managed with topical treatment. The other female patient with $\mathrm{CD}$ on combination therapy went into $\mathrm{CD}$ remission without psoriasis. After 2 years on dual therapy, she received monotherapy with UST, but had another CD flare, and was switched to adalimumab and methotrexate. Both her CD and psoriasis flared, and she was again treated with IFX and UST. After 1 year she developed plantar pustulosis psoriatic lesions and was therefore switched to VDZ monotherapy. On VDZ monotherapy she developed several small bowel strictures.

The two boys were treated for 2 years with a combination of IFX and UST. Thereafter, they were managed with monotherapy, one with UST every 8 weeks subcutaneously, the other with IFX. Both are in CD remission without psoriasis. The girl with anogenital psoriasis received combination therapy and went into clinical remission, but with endoscopic inflammation and elevated inflammatory markers. She experienced a skin infection and an external otitis that were successfully treated with antibiotics. After 2.5 years she was switched to VDZ monotherapy, initially supported by corticosteroids, and is in remission. No other adverse events were noted.

\subsection{Literature Search}

Data on combining biological agents with a different mode of action is limited. We found no publications reporting the use of a combination of biological agents (TNF antagonists, VDZ or UST) in PIBD. However, we did identify 13 publications (most of them case series) (Table 3 ) describing the use of a combination of biological agents in adult patients with IBD [21-33]. One of the studies was a randomized controlled trial (RCT) comparing IFX and IFX in combination with natalizumab (a humanized monoclonal IgG4 antibody against $\alpha 4 \beta 1$ integrin) in the treatment of active CD [24]. There was a trend toward clinical improvement with the combination and rates of infections and adverse events were comparable between the two treatment arms. The other studies are case reports including 1-15 patients, both UC and CD. In 2019, Ribaldone et al. published a pooled analysis that included seven of these reports, involving a total of 18 patients. A clinical improvement was obtained in all patients, with endoscopic improvement in 93\% without any serious adverse events [34]. In another study, three adult patients in remission received the combination of a TNF antagonist and UST for paradoxical psoriasis resistant to topical therapy 
Table 3 Publications on combination of biologicals in the treatment of inflammatory bowel disease

\begin{tabular}{|c|c|c|c|c|c|c|c|}
\hline Study & Year & Study type & Disease & $\begin{array}{l}\text { No. of } \\
\text { sub- } \\
\text { jects }\end{array}$ & Medication & Efficacy & Adverse events \\
\hline Sands et al. [24] & 2007 & RCT & $\mathrm{CD}$ & 52 & $\mathrm{NAT}+\mathrm{IFX}$ & Good & $\begin{array}{l}\text { Headache, CD exacerba- } \\
\text { tion, nausea, nasophar- } \\
\text { yngitis }\end{array}$ \\
\hline Hirten et al. [25] & 2015 & Case report & $\mathrm{CD}$ & 1 & IFX + VDZ & Benefit & No \\
\hline $\begin{array}{l}\text { Afzali and Chiorean } \\
\text { [26] }\end{array}$ & 2016 & Case report & $\mathrm{CD}$ & 1 & $\mathrm{ADA}+\mathrm{VDZ}$ & Remission & No \\
\hline Fischer et al. [29] & 2016 & Case report & $\mathrm{UC}$ & 1 & $\mathrm{CER}+\mathrm{VDZ}$ & Remission & No \\
\hline Yzet et al. [23] & 2016 & Case report & $\mathrm{CD} / \mathrm{UC} / \mathrm{PS}$ & 3 & IFX + UST & $\begin{array}{l}\text { Not effective for } \\
\text { psoriasis }\end{array}$ & No \\
\hline Bethge et al. [32] & 2017 & Case report & Pouchitis/SpA & 1 & $\mathrm{ETA}+\mathrm{VDZ}$ & Remission & No \\
\hline Liu and Loomes [28] & 2017 & Case report & $\mathrm{CD}$ & 1 & VDZ+ UST & Remission & No \\
\hline Huff-Hardy et al. [27] & 2017 & Case report & CD & 1 & $\mathrm{UST}+\mathrm{VDZ}$ & Remission & Rotavirus \\
\hline Roblin et al. [30] & 2018 & Case report & UC & 1 & $\mathrm{VDZ}+\mathrm{GOL}$ & Remission & No \\
\hline Buer et al. [21] & 2018 & Case series & $\mathrm{CD} / \mathrm{UC}$ & 10 & $\begin{array}{l}9 \mathrm{IFX}+\mathrm{VDZ} \\
1 \mathrm{ADA}+\mathrm{UST}\end{array}$ & Remission & 3 UAI \\
\hline Mao et al. [31] & 2018 & Case series & $\mathrm{CD}$ & 4 & $\begin{array}{l}1 \mathrm{ETA}+\mathrm{UST} / \mathrm{VDZ} \\
1 \mathrm{VDZ}+\mathrm{UST} \\
2 \mathrm{VDZ}+\mathrm{GOL}\end{array}$ & Remission in $3 / 4$ & $\begin{array}{l}\text { HFMD, influenza, } \\
\text { Clostridium difficile }\end{array}$ \\
\hline Elmoursi et al. [33] & 2020 & Case report & $\mathrm{CD}$ & 1 & UST + VDZ & Remission & No \\
\hline Kwapisz et al. [22] & 2020 & Case series & $\mathrm{CD} / \mathrm{UC}$ & 15 & $\begin{array}{l}8 \mathrm{VDZ}+\mathrm{TNF} \\
5 \mathrm{VDZ}+\mathrm{UST} \\
2 \mathrm{UST}+\mathrm{TNF}\end{array}$ & $\begin{array}{l}11 / 15 \text { clinical improve- } \\
\text { ment }\end{array}$ & $\begin{array}{l}\text { Salmonella, Clostridium } \\
\text { difficile, } 4 \text { infections, } \\
\text { arthralgia }\end{array}$ \\
\hline
\end{tabular}

All reports were limited to adult patients

$A D A$ adalimumab, $C D$ Crohn's disease, $C E R$ certolizumab, ETA etanercept, $G O L$ golimumab, $H F M D$ hand, foot, and mouth disease, $I F X$ infliximab, NAT natalizumab, $P S$ psoriasis, $R C T$ randomized controlled trial, $S p A$ spondyloarthritis, $T N F$ tumor necrosis factor inhibitor, $U A I$ upper airway infections, $U C$ ulcerative colitis, $U S T$ ustekinumab, $V D Z$ vedolizumab

and methotrexate [23]. The combination therapy was well tolerated but was terminated after 2 months due to lack of effect on the psoriasis. In a case series from the Mayo Clinic including 15 patients (14 CD, $1 \mathrm{UC}$ ), combination therapy with biological agents was used due to disease worsening [22]. Eleven patients had symptomatic improvement and the authors conclude that combining biologics with different mechanisms of action may be safe and effective in the treatment of IBD. Finally, there is one ongoing RCT investigating the potential effect of triple therapy with VDZ, adalimumab, and oral methotrexate on endoscopic remission in newly diagnosed adult $\mathrm{CD}$ patients at high risk for complications [ClinicalTrials.gov identifier NCT02764762].

\section{Discussion}

We have experienced improved clinical efficacy combining biologicals in selected moderate to severe PIBD patients without the occurrence of serious adverse events. The indications for this treatment strategy in our patients were partial effect of IFX and therefore a need for treatment escalation, or side effects such as paradoxical psoriasis. To our knowledge, this is the first report of combining biological agents in PIBD.

According to a recent study, patients co-exposed to antiTNF while receiving VDZ induction therapy did not experience higher rate of adverse events compared with patients exposed to VDZ alone, indicating there is no need for a waiting interval before starting VDZ in patients who recently stopped anti-TNF [35]. Combining a TNF antagonist that has a rapid systemic effect with a slower acting gut-specific agent like VDZ seems very attractive and adult experience in combining VDZ with IFX in IBD patients indicate that the combination is safe and efficacious [34]. In patients with only a partial improvement with anti-TNF, this combination may also act as a bridge to monotherapy with VDZ. In addition, corticosteroids can be avoided, which is of special interest in children [36].

Most IBD patients have inactive or mild disease activity when paradoxical psoriasis appears [37]. This was also the case in our patients. UST has been proven to be effective in treating anti-TNF-induced psoriasis and psoriasiform alopecia [35, 38, 39]. In most cases, topical treatment is sufficient, 
and few patients need to end the anti-TNF therapy. In our patients, the psoriasis was extensive, not controlled by topical treatments, and involved hair loss. A switch to monotherapy with UST was tried in two patients, resulting in a flare of CD. Maybe we would have obtained a better result if we had used an intravenous loading dose with UST, which is recommended in IBD [5, 40], but that was not available at the time. Combination therapy with UST and IFX due to paradoxical psoriasis may not be an indefinite treatment. Two of our patients have discontinued combination therapy and have stayed in clinical remission with IFX or UST monotherapy for $>3$ years without reoccurrence of psoriasis.

The combination treatments with biologicals were well tolerated in our patients. This is consistent with several published case series in adult patients [21-23]. Among our patients, one girl with CD and PSC developed severe eczema on the face and body, and in one boy with UC, elevated transaminases were detected after induction of clinical remission with VDZ and IFX combination therapy. In both patients, the treatment with IFX was stopped. The eczema was most probably due to IFX as it resolved after IFX discontinuation and the elevated transaminases were most likely related to PSC. Two patients developed antibodies to IFX but remained in remission with VDZ monotherapy. Apart from a skin infection and an external otitis in one patient, we did not notice any other adverse events.

Immunogenicity with the formation of anti-drug antibodies seems to have little or no importance when using UST or VDZ in the treatment of IBD and, in contrast to antiTNFs, co-medication with immunosuppressants is usually not recommended [41]. We stopped the use of immunosuppressants in all except two of our patients, since there is an increased risk of severe adverse events related to extensive immune suppression. Development of antibodies to IFX in two patients in whom concomitant immunosuppression was stopped was probably a consequence of this; therefore, there might still be a need for immunosuppression in patients receiving anti-TNFs as part of the combination. Such triple regimens are probably less problematic when using VDZ because of the gut-selective mode of action of this agent. However, most importantly, in every patient the risks of extensive immune suppression must be weighed against the severity of the disease.

In two of our patients, their IBD got worse when we tried monotherapy with VDZ and it was evident that they needed the combination treatment. In the other six patients who received combination with IFX, VDZ monotherapy might have induced remission. Among those patients who experienced anti-TNF-induced psoriasis, there were two patients who received monotherapy with subcutaneous UST with good effect on the skin lesions, but insufficient effect on the IBD. When switching to another class of biologics due to failure of monotherapy with IFX (and other anti-TNF drugs), monotherapy with the next biological is still our routine until RCTs have provided evidence for the opposite.

This is a small case report. The results must be interpreted with caution, especially with respect to the safety data. An obvious weakness of our study is the retrospective nature. Other therapeutic options had been tried, but not systematically in all patients. For example, as previously mentioned, monotherapy with UST or VDZ was not tried in all patients before combining biologicals. A strength of or study is that all patients treated with IFX and VDZ were well characterized by endoscopic evaluation, laboratory work-up, fecal calprotectin, drug levels, and measurements of antidrug antibodies (see Table 1) to verify inflammation and optimize ongoing therapy.

\section{Conclusions}

In patients with moderate to severe IBD who lose response to an anti-TNF agent or develop side effects such as paradoxical psoriasis, combining the anti-TNF with another biological agent with a different mode of action may be beneficial. We did not experience any severe adverse events when combining IFX with VDZ or UST in PIBD patients. Our findings should, however, be addressed in larger studies with a long-term follow-up.

Acknowledgments Open Access funding provided by Akershus University Hospital (AHUS).

Author contributions All authors have made substantial contributions to all of the following: (1) the conception and design of the study, or acquisition of data, or analysis and interpretation of data, (2) drafting the article or revising it critically for important intellectual content, (3) final approval of the version to be submitted.

\section{Compliance with Ethical Standards}

Conflict of interest $\mathrm{CO}$ has served as a speaker, consultant, or advisory board member for AbbVie, Nutricia, Norgine, Tillotts Pharma, and Mead Johnson. JJ has served as a speaker, consultant, or advisory board member for Abbie, Astro Pharma, Boehringer Ingelheim, BMS, Ferring, Celltrion, Hikma, Janssen, Meda, MSD, Napp Pharma, Norgine, Novartis, Orion Pharma, Pfizer, Pharmacosmos, Roche, Takeda, Tillotts, and Sandoz. JBR has no conflicts of interest to disclose.

Ethics approval The study was approved as a quality assurance study by the Data Protection Official for Research at Akershus University Hospital and good clinical practice guidelines were followed. Written informed consent is not required in quality assurance studies.

Open Access This article is licensed under a Creative Commons Attribution-NonCommercial 4.0 International License, which permits any non-commercial use, sharing, adaptation, distribution and reproduction in any medium or format, as long as you give appropriate credit to the original author(s) and the source, provide a link to the Creative 
Commons licence, and indicate if changes were made. The images or other third party material in this article are included in the article's Creative Commons licence, unless indicated otherwise in a credit line to the material. If material is not included in the article's Creative Commons licence and your intended use is not permitted by statutory regulation or exceeds the permitted use, you will need to obtain permission directly from the copyright holder.To view a copy of this licence, visit http://creativecommons.org/licenses/by-nc/4.0/.

\section{References}

1. Grover Z. Predicting and preventing complications in children with inflammatory bowel disease. Transl Pediatr. 2019;8(1):70-6.

2. Tarnok A, Kiss Z, Kadenczki O, Veres G. Characteristics of biological therapy in pediatric patients with Crohn's disease. Exp Opin Biolog Ther. 2019;19(3):181-96.

3. Sridhar S, Maltz RM, Boyle B, Kim SC. Dermatological manifestations in pediatric patients with inflammatory bowel diseases on anti-TNF therapy. Inflamm Bowel Dis. 2018;24:2086-92.

4. Sandborn WJ, Gasink C, Gao LL, Blank MA, Johanns J, Guzzo C, et al. Ustekinumab induction and maintenance therapy in refractory Crohn's disease. N Engl J Med. 2012;367(16):1519-28.

5. Feagan BG, Rutgeerts P, Sands BE, Hanauer S, Colombel JF, Sandborn WJ, et al. Vedolizumab as induction and maintenance therapy for ulcerative colitis. N Engl J Med. 2013;369(8):699-710.

6. Shim HH, Chan PW, Chuah SW, Schwender BJ, Kong SC, Ling $\mathrm{KL}$. A review of vedolizumab and ustekinumab for the treatment of inflammatory bowel diseases. J Gastroenterol Hepatol Open. 2018;2(5):223-34.

7. Wyant T, Fedyk E, Abhyankar B. An overview of the mechanism of action of the monoclonal antibody vedolizumab. J Crohn's \& colitis. 2016;10(12):1437-44.

8. Singh N, Rabizadeh S, Jossen J, Pittman N, Check M, Hashemi $\mathrm{G}$, et al. Multi-center experience of vedolizumab effectiveness in pediatric inflammatory bowel disease. Inflamm Bbowel Dis. 2016;22(9):2121-6.

9. Conrad MA, Stein RE, Maxwell EC, Albenberg L, Baldassano RN, Dawany N, et al. Vedolizumab therapy in severe pediatric inflammatory bowel disease. Inflamm Bowel Dis. 2016;22(10):2425-31.

10. Schneider AM, Weghuber D, Hetzer B, Entenmann A, Muller T, Zimmermann G, et al. Vedolizumab use after failure of TNF-alpha antagonists in children and adolescents with inflammatory bowel disease. BMC Gastroenterol. 2018;18(1):140.

11. Hamel B, Wu M, Hamel EO, Bass DM, Park KT. Outcome of tacrolimus and vedolizumab after corticosteroid and anti-TNF failure in paediatric severe colitis. BMJ Open Gastroenterol. 2018;5(1):e000195.

12. Ledder O, Assa A, Levine A, Escher JC, de Ridder L, Ruemmele $\mathrm{F}$, et al. Vedolizumab in paediatric inflammatory bowel disease: a retrospective multi-centre experience from the paediatric IBD Porto Group of ESPGHAN. J Crohn's Colitis. 2017;11(10):1230-7.

13. Russell RK, Hansen R, Turner D. New treatments for ulcerative colitis: do we have pediatric data? Exp Rev Clin Immunol. 2016;12(7):701-4.

14. Landells I, Marano C, Hsu MC, Li S, Zhu Y, Eichenfield LF, et al. Ustekinumab in adolescent patients age 12 to 17 years with moderate-to-severe plaque psoriasis: results of the randomized phase 3 CADMUS study. J Amer Acad Dermatol. 2015;73(4):594-603.

15. Feagan BG, Sandborn WJ, Gasink C, Jacobstein D, Lang Y, Friedman JR, et al. Ustekinumab as induction and maintenance therapy for Crohn's disease. New Engl J Med. 2016;375(20):1946-60.
16. Sands BE, Sandborn WJ, Panaccione R, O'Brien CD, Zhang $\mathrm{H}$, Johanns $\mathrm{J}$, et al. Ustekinumab as induction and maintenance therapy for ulcerative colitis. New Engl J Med. 2019;381(13):1201-14.

17. Bishop C, Simon H, Suskind D, Lee D, Wahbeh G. Ustekinumab in pediatric Crohn disease patients. J Pediatr Gastroenterol Nutr. 2016;63(3):348-51.

18. Dayan JR, Dolinger M, Benkov K, Dunkin D, Jossen J, Lai J, et al. Real world experience with ustekinumab in children and young adults at a tertiary care pediatric inflammatory bowel disease center. J Pediatr Gastroenterol Nutr. 2019;69:61-7.

19. Chavannes M, Martinez-Vinson C, Hart L, Kaniki N, Chao CY, Lawrence $\mathrm{S}$, et al. Management of paediatric patients with medically refractory Crohn's disease using ustekinumab: a multi-centred cohort study. J Crohn's Colitis. 2019;13(5):578-84.

20. van Hoeve K, Dreesen E, Hoffman I, Van Assche G, Ferrante M, Gils A, et al. Higher infliximab trough levels are associated with better outcome in paediatric patients with inflammatory bowel disease. J Crohn's Colitis. 2018;12(11):1316-25.

21. Buer LCT, Hoivik ML, Warren DJ, Medhus AW, Moum BA. Combining anti-TNF-alpha and vedolizumab in the treatment of inflammatory bowel disease: a case series. Inflamm Bowel Dis. 2018;24(5):997-1004.

22. Kwapisz L, Raffals LE, Bruining DH, Pardi DS, Tremaine WJ, Kane SV, et al. Combination biologic therapy in inflammatory bowel disease: experience from a tertiary care center. Clin Gastroenterol Hepatol. 2020 (in press).

23. Yzet C, Dupas JL, Fumery M. Ustekinumab and anti-TNF combination therapy in patients with inflammatory bowel disease. Am J Gastroenterol. 2016;111(5):748-9.

24. Sands BE, Kozarek R, Spainhour J, Barish CF, Becker S, Goldberg L, et al. Safety and tolerability of concurrent natalizumab treatment for patients with Crohn's disease not in remission while receiving infliximab. Inflamm Bowel Dis. 2007;13(1):2-11.

25. Hirten R, Longman RS, Bosworth BP, Steinlauf A, Scherl E. Vedolizumab and infliximab combination therapy in the treatment of Crohn's disease. Am J Gastroenterol. 2015;110(12):1737-8.

26. Afzali A, Chiorean M. Combination of biologic agents in the management of severe refractory Crohn's disease: a case report of concomitant treatment with vedolizumab and adalimumab: 1725 . Am J Gastroenterol. 2016;111:S823-S824824.

27. Huff-Hardy K, Bedair M, Vazquez R, Burstein E. Efficacy of combination vedolizumab and ustekinumab for refractory Crohn's disease. Inflamm Bowel Dis. 2017;23(10):E49.

28. Liu EY, Loomes DE. Ustekinumab and vedolizumab dual biologic therapy in the treatment of Crohn's disease. Case Rep Med. 2017;2017:5264216.

29. Fischer S, Rath T, Geppert CI, Manger B, Schett G, Neurath MF, et al. Long-term combination therapy with anti-TNF plus vedolizumab induces and maintains remission in therapy-refractory ulcerative colitis. Am J Gastroenterol. 2017;112(10):1621-3.

30. Roblin X, Paul S, Ben-Horin S. Co-treatment with golimumab and vedolizumab to treat severe UC and associated spondyloarthropathy. J Crohn's Colitis. 2018;12(3):379-80.

31. Mao EJ, Lewin S, Terdiman JP, Beck K. Safety of dual biological therapy in Crohn's disease: a case series of vedolizumab in combination with other biologics. BMJ Open Gastroenterol. 2018;5(1):e000243.

32. Bethge J, Meffert S, Ellrichmann M, Conrad C, Nikolaus S, Schreiber S. Combination therapy with vedolizumab and etanercept in a patient with pouchitis and spondylarthritis. BMJ Open Gastroenterol. 2017;4(1):e000127.

33. Elmoursi A, Perry C, Barrett T. Deep remission with double biologic therapy: a successful case of combination ustekinumab and vedolizumab for severe refractory Crohn's disease. Gastroenterology. 2020;158(3):S117-S11818. 
34. Ribaldone DG, Pellicano R, Vernero M, Caviglia GP, Saracco GM, Morino M, et al. Dual biological therapy with anti-TNF, vedolizumab or ustekinumab in inflammatory bowel disease: a systematic review with pool analysis. Scand J Gastroenterol. 2019;54(4):407-13.

35. Ben-Horin S, Zhao Y, Guo J, Mao R, Novack L, Sergienko R, et al. Efficacy of biological drugs in short-duration versus longduration inflammatory bowel disease: a protocol for a systematic review and an individual-patient level meta-analysis of randomised controlled trials. BMJ Open. 2019;9(1):e024222.

36. Rutgeerts PJ. Review article: the limitations of corticosteroid therapy in Crohn's disease. Alim Pharmacol Ther. 2001;15(10):1515-25.

37. Melo FJ, Magina S. Clinical management of Anti-TNF-alphainduced psoriasis or psoriasiform lesions in inflammatory bowel disease patients: a systematic review. Int J Dermatol. 2018;57(12):1521-32.
38. Tillack C, Ehmann LM, Friedrich M, Laubender RP, Papay P, Vogelsang $\mathrm{H}$, et al. Anti-TNF antibody-induced psoriasiform skin lesions in patients with inflammatory bowel disease are characterised by interferon-gamma-expressing Th1 cells and IL-17A/ IL-22-expressing Th17 cells and respond to anti-IL-12/IL-23 antibody treatment. Gut. 2014;63(4):567-77.

39. Eickstaedt JB, Killpack L, Tung J, Davis D, Hand JL, Tollefson MM. Psoriasis and psoriasiform eruptions in pediatric patients with inflammatory bowel disease treated with anti-tumor necrosis factor alpha agents. Pediatr Dermatol. 2017;34(3):253-60.

40. Sandborn WJ, Feagan BG, Rutgeerts P, Hanauer S, Colombel $\mathrm{JF}$, Sands BE, et al. Vedolizumab as induction and maintenance therapy for Crohn's disease. N Engl J Med. 2013;369(8):711-21.

41. Tamilarasan AG, Cunningham G, Irving PM, Samaan MA. Recent advances in monoclonal antibody therapy in IBD: practical issues. Frontline Gastroenterol. 2019;10(4):409-16. 